\title{
Regiospecific Chlorination of (S)- $\beta$-Tyrosyl-S-Carrier Protein Catalyzed by SgcC3 in the Biosynthesis of the Enediyne Antitumor Antibiotic C-1027
}

\author{
Shuangjun Lin ${ }^{1}$, Steven G. Van Lanen ${ }^{1}$, and Ben Shen ${ }^{1,2,3,{ }^{*}}$ \\ 1 Division of Pharmaceutical Sciences \\ 2 University of Wisconsin National Cooperative Drug Discovery Group \\ 3Department of Chemistry, University of Wisconsin-Madison, Madison, WI 53705
}

\begin{abstract}
C-1027 is a potent antitumor antibiotic composed of an apo-protein and a reactive enediyne chromophore. The chromophore consists of four different chemical subunits including an (S)-3chloro-4,5-dihydroxy- $\beta$-phenylalanine moiety, the biosynthesis of which from L- $\alpha$-tyrosine is catalyzed by six proteins, $\mathrm{SgcC}, \mathrm{SgcC} 1, \mathrm{SgcC} 2, \mathrm{SgcC} 3, \mathrm{SgcC} 4$, and $\mathrm{SgcC} 5$. Biochemical characterization of $\mathrm{SgcC} 3$ unveiled that: (i) $\mathrm{SgcC} 3$ is a flavin adenine dinucleotide (FAD)-dependent halogenase, (ii) $\mathrm{SgcC} 3$ acts only on the $\mathrm{SgcC} 2$ peptidyl carrier protein-tethered substrates, (iii) $\mathrm{SgcC} 3$-catalyzed halogenation requires $\mathrm{O}_{2}$ and reduced $\mathrm{FAD}$ and either the $\mathrm{C}-1027$ pathway-specific flavin reductase $\mathrm{SgcE} 6$ or $E$. coli flavin reductase (Fre) can support the $\mathrm{SgcC} 3$ activity, (iv) $\mathrm{SgcC} 3$ also efficiently catalyzes bromination but not fluorination or iodination, and (v) $\mathrm{SgcC} 3$ can utilize both $(S)$ - and $(R)$ - $\beta$-tyrosyl-S-SgcC2 but not 3-hydroxy- $\beta$-tyrosyl-S-SgcC2 as a substrate. These results establish that $\mathrm{SgcC} 3$ catalyzes the third enzymatic transformation during the biosynthesis of the (S)-3-chloro-4,5-dihydroxy- $\beta$-phenylalanine moiety moiety of C- 1027 from L- $\alpha$-tyrosine. SgcC3 now represents the second biochemically characterized flavin-dependent halogenase that acts on a carrier protein-tethered substrate. These findings will facilitate the engineering of new C-1027 analogs by combinatorial biosynthesis methods.
\end{abstract}

\section{Keywords}

C-1027; chlorination; enediyne; halogenase; SgcC3; Streptomyces globisporus

\section{Introduction}

C-1027 is a chromoprotein antitumor antibiotic isolated from the fermentation broth of Streptomyces globisporus. ${ }^{1}$ Similar to other nine-membered enediynes, which includes neocarzinostatin ${ }^{2}$ and maduropeptin, ${ }^{3} \mathrm{C}-1027$ is composed of an apo-protein (CagA) and a reactive enediyne chromophore that is essential for bioactivity. ${ }^{4}$ Upon release from CagA, the C-1027 chromophore readily undergoes a Bergman cycloaromatization to form a benzenoid diradical intermediate (Figure 1). These free radicals are capable of abstracting hydrogen atoms from the DNA backbone, ultimately resulting in DNA double-stranded breaks (DSB) in the presence of molecular oxygen. ${ }^{5-7}$ The induction of DSB correlates well with cytotoxicity, and 
because of this novel mechanism of DNA damage, the enediynes have attracted interest as potential cancer chemotherapeutic agents. 8,9

The nine-membered enediynes all have an enediyne core consisting of an unsaturated ninemembered carbocycle containing two acetylenic groups conjugated to a recipient or incipient double bond. The C-1027 chromophore (1) has three additional chemical components that are covalently appended to the enediyne core, namely a deoxy aminosugar, a benzoxazolinate, and a $\beta$-amino acid moiety (Figure 1 ). The $\mathrm{C}-1027$ biosynthetic gene cluster was previously identified and, based on bioinformatics analysis, the biosynthetic pathway for each moiety was predicted and a convergent pathway was hypothesized to yield 1 . Of note, the $\beta$-amino acid moiety $(S)$-3-chloro-4,5-dihydroxy- $\beta$-phenylalanine was predicted to be biosynthesized from L- $\alpha$-tyrosine (2) mediated by five proteins: $\mathrm{SgcC}, \mathrm{SgcC} 1, \mathrm{SgcC} 2, \mathrm{SgcC} 3$ and $\mathrm{SgcC} 4$, with the condensation enzyme SgcC5 catalyzing the final incorporation of $\mathbf{2}$ into $\mathbf{1}$ via a $\beta$-aminoacyl$\mathrm{S}$-SgcC2 intermediate (3) (Figure 2). ${ }^{8}$ We have subsequently confirmed in vitro using purified recombinant enzymes that (i) SgcC4 catalyzes the conversion of $\mathbf{2}$ to $(S)$ - $\beta$-tyrosine (4) as the first step ${ }^{10-12}$ and (ii) $\mathrm{SgcC} 1$ activates 4 as an $(S)$ - $\beta$-tyrosyl adenylate (5) and subsequently loads 4 to $\mathrm{SgcC} 2$, a peptidyl carrier protein (PCP), to yield $(S)$ - $\beta$-tyrosyl-S-SgcC2 $(\mathbf{6})$ as the second step. ${ }^{13,14} \mathrm{SgcC} 3$ and $\mathrm{SgcC}$ were also identified as the C-3 halogenase and C-5 hydroxylase, respectively, by gene inactivation: the $\triangle \operatorname{sgcC} 3$ mutant produced 20-deschloro$\mathrm{C}-1027$ (7) and 20-deschloro-22-deshydroxy-C-1027 (8), ${ }^{13}$ while the $\Delta s g c C$ mutant accumulated 22-deshydroxy-C-1027 (9). ${ }^{8}$ Thus, the requirement of $\mathrm{SgcC} 3$ for chlorination was clearly established, although these results provided little insight into the substrate specificities and mechanistic details of this enzyme.

In general, incorporation of a halogen into natural products plays an important role in increasing the diversity and biological activity of natural products. Indeed, 7 was recently shown to be less proficient at inducing DSB, less cytotoxic, as well as have an altered mechanism of action compared to the typical cellular response induced by treatment with 1. 9 Prior to 1997 , halogenation was believed to occur only by the action of haloperoxidases - enzymes that utilize a heme or vanadium cofactor and hydrogen peroxide as a co-substrate. ${ }^{15}$ Since this time, several $\mathrm{O}_{2}$-dependent halogenases have been described and their activity biochemically confirmed. Currently, the $\mathrm{O}_{2}$-dependent halogenases fall into one of two families: $\alpha$ ketoglutarate-dependent halogenases that act on non-activated aliphatic substrates $16-21$ and flavin-dependent halogenases that catalyze the regioselective halogenation of primarily aromatic compounds. $16,22-31 \mathrm{SgcC} 3$ has sequence homology to flavin-dependent halogenases, and combined with the in vivo data, $\mathrm{SgcC} 3$ was predicted to incorporate chloride using flavin as a redox cofactor and $\mathrm{O}_{2}$ as an oxidizing agent.

Given the importance of halogen atoms in natural products and the intriguing variety and complexity of halogenases, ${ }^{16}$ we set out to characterize $\mathrm{SgcC} 3$ in vitro as part of our continuous efforts to investigate the biosynthetic pathway of $\mathbf{1}$. In this study, we report the functional characterization of recombinant $\mathrm{SgcC} 3$ as a flavin adenine dinucleotide (FAD)-dependent halogenase that catalyzes the regiospecific chlorination of $\mathbf{6}$, requiring $\mathrm{O}_{2}$ and reduced FAD $\left(\mathrm{FADH}_{2}\right)$, to afford $(S)$-3-chloro- $\beta$-tyrosyl-S-SgcC2 $(\mathbf{1 0})$ as the third step for the biosynthesis of 3 from 2 (Figure 2). The general catalytic properties of $\mathrm{SgcC} 3$ are reported, and $\mathrm{SgcC} 3$ now is the second biochemically characterized flavin-dependent halogenase that acts on a carrier protein-tethered substrate, which likely represents a general strategy for oxidative halogenation of secondary metabolites that are assembled via carrier protein-dependent biosynthetic machinery. The results established herein, along with the evidence that SgcC5 has relaxed specificity as implied by the isolation of $\mathbf{7 , 8}$, and $\mathbf{9}$, affords the opportunity to generate new C-1027 analogs by combinatorial biosynthesis methods. 


\section{Experimental Procedures}

\section{Synthesis of the $\beta$-Tyrosine Analogues}

Syntheses of the $\beta$-tyrosine analogues [i.e., 3 -chloro- $\beta$-tyrosine, 3 -bromo- $\beta$-tyrosine, 3 hydroxy- $\beta$-tyrosine, and 3 -chloro-5-hydroxy- $\beta$-tyrosine] were achieved by following literature procedure $^{32}$ (see Supporting Information for details).

\section{Cloning of sgcC2, sgcC3, sgcE6, and E. coli fre Genes for Heterologous Expression}

The genes encoding $\mathrm{SgcC} 2, \mathrm{SgcC} 3, \mathrm{SgcE} 6$ and E. coli flavin reductase (Fre) were amplified by PCR using Platinum Pfx DNA polymerase following the program and conditions provided by Invitrogen (see Table S1 for primers used in Supporting Information). Templates utilized

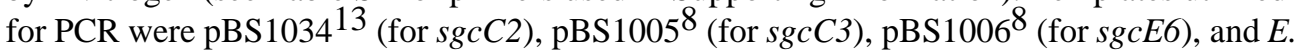
coli DH5 $\alpha$ genomic DNA (for fre), respectively. Purified PCR product of $s g c C 2$ was cloned into the $\mathrm{pCDF}-2 \mathrm{Ek} / \mathrm{LIC}$ vector using ligation-independent cloning procedure as described by Novagen (Madison, WI) to give pCDF-2Ek/LIC-SgcC2 (pBS1040). The PCR products of $s g c C 3, s g c E 6$ and fre were similarly cloned into the pET-30Xa/LIC vector (Novagen, Madison, WI) to yield pET-30Xa/LIC-SgcC3 (pBS1041), pET-30Xa/LIC-SgcE6 (pBS1042), and pET-30XaLIC-Fre (pBS1043), respectively. All cloned PCR products were confirmed by DNA sequencing. The $s g c C l$ expression construct pBS1033 has been described previously. ${ }^{13}$

\section{Overproduction and Purification of SgcC1, SgcC2, SgcC3, SgcE6 and Fre}

E. coli BL21 (DE3) introduced with pBS1033 (for SgcC1), pBS1040 (for SgcC2), pBS1041 (for $\mathrm{SgcC} 3$ ), pBS1042 (SgcE6), or pBS1043 (for Fre) was cultured in LB medium ${ }^{33}$ supplemented with streptomycin $\left(50 \mu \mathrm{g} \mathrm{mL}^{-1}\right.$ for $\left.\mathrm{pBS} 1040\right)$ or kanamycin $\left(50 \mu \mathrm{g} \mathrm{mL} \mathrm{L}^{-1}\right.$ for pBS1033, pBS1041, pBS1042, pBS1043), respectively. All cells were grown at $18^{\circ} \mathrm{C}$ and induced with IPTG (final concentration of $0.1 \mathrm{mM}$ ) when $\mathrm{OD}_{600}$ reached $\sim 0.5$. They were further cultured at $18{ }^{\circ} \mathrm{C}$ for additional $15 \mathrm{hrs}$. Cells were harvested by centrifugation $\left(4^{\circ} \mathrm{C}\right.$, $8000 \mathrm{rpm}$ for $15 \mathrm{~min})$ and resuspended in Buffer A (100 mM sodium phosphate, $\mathrm{pH} 7.5$, containing $300 \mathrm{mM} \mathrm{NaCl}$ ) supplemented with a complete protease inhibitor tablet, EDTA-free (Roche Applied Science, Indianapolis, IN). The cells were lysed by sonication $(4 \times 30 \mathrm{sec}$ pulsed cycle), and the debris was removed by centrifugation $\left(4^{\circ} \mathrm{C}, 15000 \mathrm{rpm}\right.$ for $50 \mathrm{~min}$ ). The clarified supernatant was loaded onto a pre-equilibrated Ni-NTA agarose column (Qiagen, Valencia, CA) with Buffer B (Buffer A plus 10\% glycerol). The column was washed with 5column volumes of Buffer B followed by 5-column volumes of Buffer B containing $20 \mathrm{mM}$ imidazole. The $\mathrm{His}_{6}$-tagged proteins were eluted with 6-column volumes of Buffer B containing $250 \mathrm{mM}$ imidazole. $\mathrm{SgcC} 2$ was dialyzed in $50 \mathrm{mM}$ Tris- $\mathrm{HCl}, \mathrm{pH} 7.5,50 \mathrm{mM} \mathrm{NaCl}$ and $1 \mathrm{mM}$ dithiothreitol. $\mathrm{SgcC} 1, \mathrm{SgcC} 3, \mathrm{SgcE} 6$, and Fre were desalted using a PD-10 column (GE Healthcare, Piskataway, NJ). After concentration with an Amicon Ultra-4 (5K or 10K), the purified proteins were stored at $-25{ }^{\circ} \mathrm{C}$ in $40 \%$ glycerol. Protein purity was assessed as > $90 \%$ by $12-15 \%$ SDS-PAGE. Protein concentrations were determined using the Bradford assay (Bio-Rad, Hercules, CA)

\section{Determination of the SgcC3 Flavin Cofactor}

$\mathrm{SgcC} 3$ was denatured by heat or with 50\% methanol (final concentration) to release any noncovlently-bound cofactor. After centrifugation the supernatent was analyzed on C18 reverse phase column $(250 \times 4.6 \mathrm{~mm}$, Alltech Associates Inc. Deerfield, IL) using a linear gradient from 0 to $60 \%$ acetonitrile (in water) at a flow rate of $1 \mathrm{~mL} \mathrm{~min}^{-1}$ with detection at $266 \mathrm{~nm}$. 


\section{Enzymatic Synthesis of $\beta$-Aminoacyl-S-SgcC2}

Post-translational modification of apo-SgcC2 into holo- $\mathrm{SgcC} 2$ was achieved in a reaction mixture containing $100 \mathrm{mM}$ Tris- $\mathrm{HCl}, \mathrm{pH} 7.5,160 \mu \mathrm{M}$ apo-SgcC2, $0.8 \mathrm{mM}$ coenzyme A (CoA), $12.5 \mathrm{mM} \mathrm{MgCl} 2,2.0 \mathrm{mM}$ tris(2-carboxyethyl)phosphine hydrochloride (TCEP), and $5 \mu \mathrm{M}$ Svp. ${ }^{34}$ After incubation at room temperature for $45 \mathrm{~min}$, an equal volume of loading solution consisting of $2 \mathrm{mM} 4$ [ $4 \mathrm{mM}$ for $(R)$ - $\beta$-tyrosine (11) or 3-hydroxy- $\beta$-tyrosine], $4 \mathrm{mM}$ adenosine triphosphate, $2.0 \mathrm{mM}$ TCEP and $12.5 \mathrm{mM} \mathrm{MgCl}_{2}$ (final concentration in the loading reaction mixture) was added. Amino acid loading was initiated by the addition of $\mathrm{SgcCl}$ to a final concentration of $2 \mu \mathrm{M}$ ( $6 \mu \mathrm{M}$ for $\mathbf{1 1}$ or 3 -hydroxy- $\beta$-tyrosine), and the resulting solution was incubated at room temperature for an additional $60 \mathrm{~min}$ as described previously. ${ }^{13}$ The resulting $\beta$-aminoacyl-S-SgcC2 substrates [i.e., 6, $(R)$ - $\beta$-tyrosyl-S-SgcC2 (12), or 3-hydroxy$\beta$-tyrosyinl-S-SgcC2] were purified from loading mixture with a 5-mL HiTrap Q anionexchange column (GE Healthcare, Piskataway, NJ). The column was pre-equilibrated with 20 $\mathrm{mM}$ sodium phosphate buffer, $\mathrm{pH} 7.0$, and the crude $\mathrm{SgcC} 2$-tethered substrate preparations were loaded and eluted using a linear gradient of 0 to $100 \%$ of $1 \mathrm{M} \mathrm{NaCl}$ for 25 -column volumes and flow rate of $3 \mathrm{~mL} \mathrm{~min}{ }^{-1}$. The purified $\beta$-aminoacyl-S-SgcC2 substrates, which were eluted between $0.35 \mathrm{M}$ and $0.4 \mathrm{M} \mathrm{NaCl}$, were desalted using size-exclusion chromatography (Superose ${ }^{\circledR}$ 12, GE Healthcare, Piskataway, NJ) and concentrated prior to use in $\mathrm{SgcC} 3$ assays.

\section{In vitro Activity Assay for SgcC3}

The $\mathrm{SgcC} 3$ assay solution contained $50 \mu \mathrm{M} 6$ (or 12 or $250 \mu \mathrm{M}$ 3-hydroxy- $\beta$-tyrosyl-S-SgcC2), $5 \mathrm{mM} \beta$-nicotinamide adenine dinuceotide, reduced (NADH), $0.10 \mathrm{mM} \mathrm{FAD}, 100 \mathrm{mM} \mathrm{NaCl}$, $1 \mathrm{mM}$ TCEP, $20 \mu \mathrm{M} \mathrm{SgcC} 3$, and $5 \mu \mathrm{M}$ SgcE6 in $50 \mathrm{mM}$ sodium phosphate buffer, $\mathrm{pH}$ 6.0. Reactions were incubated at $37^{\circ} \mathrm{C}$ for $1 \mathrm{hr}$. The reaction was terminated by the addition of 70 $\%$ trichloroacetic acid (TCA) to a final concentration of $10 \%$ to precipitate all proteins. After incubation on ice for $15 \mathrm{~min}$, the precipitate was separated by centrifugation $\left(4^{\circ} \mathrm{C}, 14,000 \mathrm{rpm}\right.$ for $15 \mathrm{~min}$ ). The resulting pellet was washed twice with $200 \mu \mathrm{L}$ of $5 \%$ TCA and once with 200 $\mu \mathrm{L}$ of ethanol. After drying by speed-vac, the protein pellet was re-dissolved in $150 \mu \mathrm{L}$ of 0.1 $\mathrm{M} \mathrm{KOH}$ and incubated at $70^{\circ} \mathrm{C}$ for 15 min to hydrolyze all $\mathrm{SgcC} 2$-tethered $\beta$-amino acids. After removal of the proteins by centrifugation, the solution was concentrated by speed-vac and analyzed for 4 (or $\mathbf{1 1}$ or 3-hydroxy- $\beta$-tyrosine) and the expected product ( $S$ )-3-chloro- $\beta$ tyrosine (13) [or $(R)$-3-chloro- $\beta$-tyrosine (14) or 3-chloro-5-hydroxy- $\beta$-tyrosine] by a Varian ProStar 210 HPLC System equipped with a C18 reverse-phase column $(250 \times 4.6 \mathrm{~mm}$, Alltech Associates Inc., Deerfield, IL), using a 24-min linear gradient from 0 to $40 \%$ (25\% for 3hydroxy- $\beta$-tyrosine and 3 -chloro-5-hydroxy- $\beta$-tyrosine) acetonitrile ( $0.1 \%$ TFA) at a flow rate of $1 \mathrm{~mL} \mathrm{m^{-1 }}$ and detection at $280 \mathrm{~nm}$ and authentic 4, 11, 13, 14, 3-hydroxy- $\beta$-tyrosine, or 3 -chloro-5-hydroxy- $\beta$-tyrosine as references. Control experiments were carried out under identical conditions but with boiled $\mathrm{SgcC} 3$.

\section{Formation of 3-Bromo- $\beta$-Tyrosyl-S-SgcC2}

Both $\mathrm{SgcC} 3$ and SgcE6 were prepared by purification using Ni-NTA columns in buffers without chloride. After 4 was loaded onto holo-SgcC2 by $\mathrm{SgcC} 1$, the resultant product 6 was purified from the loading reaction mixture through a 5-mL HiTrap Q column. Chloride was then completely removed by passing through a size-exclusion column twice (Superose ${ }^{\circledR} 12$ ). The SgcC3-catalyzed bromination of $\mathbf{6}$ was performed in a solution identical to above except that $0.1 \mathrm{M} \mathrm{NaBr}$ replaced $\mathrm{NaCl}$ in the assay mixture and TCEP was excluded. The reaction was quenched with $10 \%$ TCA to precipitate proteins at 10,30 and $60 \mathrm{~min}$ as described above. The precipitate was treated with $150 \mu \mathrm{L}$ of $0.1 \mathrm{M} \mathrm{KOH}$ to release all SgcC2-tethered $\beta$-amino acids. The resultant free $\beta$-amino acids [i.e., 4 and the expected product 3 -bromo- $\beta$-tyrosine (15)] were subjected to HPLC and MS analysis. The standard curve was calibrated with 
synthetic 15 to correlate peak area with product amount formed in each reaction. The product formation was fitted into a linear equation to obtain the initial velocity.

\section{Initial Velocity of SgcC3 with (S)- $\beta$-Tyrosyl-S-SgcC2 and (R)- $\beta$-Tyrosyl-S-SgcC2}

Conversion of apo- $\mathrm{SgcC} 2$ to holo- $\mathrm{SgcC} 2$ was carried out in a $1.8 \mathrm{~mL}$ reaction containing 400 $\mu \mathrm{M} \mathrm{SgcC} 2,1.6 \mathrm{mM} \mathrm{CoA}$, and $10 \mu \mathrm{M} \mathrm{Svp}$ at room temperature for $60 \mathrm{~min}$. For preparation of 6 from 4, the loading condition was identical to the standard assay condition described above. For preparation of $\mathbf{1 2}$ from 11, the reaction proceeded with $4 \mathrm{mM} 11$ and $6 \mu \mathrm{M} \mathrm{SgcC} 1$ and was incubated at room temperature for $90 \mathrm{~min}$. Chlorination assays were performed in $200 \mu \mathrm{L}$ of reaction mixture containing $200 \mu \mathrm{M} 6$ or $\mathbf{1 2}, 0.1 \mathrm{M} \mathrm{NaCl}, 5 \mathrm{mM}$ NADH, $0.1 \mathrm{mM}$ FAD, 25 $\mu \mathrm{M} \mathrm{SgcC} 3$, and $3 \mu \mathrm{M} \mathrm{SgcE6}$. The reaction was quenched by the addition of $30 \mu \mathrm{l}$ of $70 \% \mathrm{TCA}$ at 10,20, 40 and $60 \mathrm{~min}$ and analyzed as described above to determine the rate for $(S)$-3-chloro$\beta$-tyrosyl-S-SgcC2 (16) or ( $R$ )-3-chloro- $\beta$-tyrosyl-S-SgcC2 (17) formation. A standard calibration curve was created with synthetic $\mathbf{1 3}$, and product formation was fitted to a linear equation to obtain the initial velocity. The specific activity was calculated from the initial velocity divided by the concentration of $\mathrm{SgcC} 3$ as determined using the Bradford dye-binding procedure.

\section{Results}

\section{Overproduction and Purification of SgcC1, SgcC2, SgcC3, SgcE6, and Fre}

The $\operatorname{sgcC} 2$ gene was subcloned from pBS1034 ${ }^{13}$ into $\mathrm{pCDF}-2 \mathrm{Ek} / \mathrm{LIC}$ vector to eliminate most of the extra $\mathrm{N}$-terminal residues engineered into the recombinant protein used in previous studies. ${ }^{13,14}$ After overproduction in E. coli, $\mathrm{SgcC} 2$ was purified to homogeneity as an Nterminal His $_{6}$-tagged fusion protein containing an additional 13 amino acids. Purification of $\mathrm{SgcC} 3$ to homogeneity afforded a yellow solution whose UV-Vis spectrum showed the characteristic absorption maxima of $375 \mathrm{~nm}$ and $450 \mathrm{~nm}$, indicative of the presence of a flavin prosthetic group. Since it has been previously reported that $\mathrm{FADH}_{2}$-dependent halogenases require a separate flavin reductase to supply flavin cofactor in $\mathrm{FADH}_{2}$ form, ${ }^{22-31}$ the putative flavin reductase SgcE6 within the C-1027 biosynthetic gene cluster (Figure 2A) and E. coli Fre were individually overproduced and purified to homogeneity. Similar to $\mathrm{SgcC} 3$, the purified SgcE6 and Fre also had UV-Vis profiles characteristic of flavoproteins. SgcC1 was overproduced, purified, and the activity accessed as previously described. ${ }^{13,14}$ The homogeneity of the purified proteins was confirmed by SDS-PAGE analysis (Figure 3).

\section{Identity of the SgcC3 Flavin Cofactor}

As predicted from bioinformatics analysis, the purified $\mathrm{SgcC} 3$ had a UV-vis spectrum reminiscent of a flavin cofactor. Heat denaturation of $\mathrm{SgcC} 3$ completely released the flavin group, indicating that the cofactor is noncovalently bound to SgcC3. HPLC analysis of the released flavin prosthetic group with authentic FMN and FAD as standards established its identity as FAD with a 1:0.18 molar ratio of SgcC3: FAD (see Figure S1 in Supporting Information).

\section{Substrate Preparation and Activity Assay of SgcC3 with (S)- $\beta$-Tyrosyl-S-SgcC2}

Holo-SgcC2 was generated enzymatically using the Svp phosphopantetheinyl transferase (PPTase). ${ }^{34}$ Subsequently, the 4 -specific adenylation enzyme $\mathrm{SgcC} 1$ was used to load holo$\mathrm{SgcC} 2$ with 4 to generate $\mathbf{6}$. After purification using anion exchange, $\mathbf{6}$ was incubated with $\mathrm{NaCl}$ in the presence of $\mathrm{SgcC} 3, \mathrm{SgcE} 6, \mathrm{FAD}$, and $\mathrm{NADH}$ under aerobic condition at $37^{\circ} \mathrm{C}$ for $1 \mathrm{hr}$, and the reaction was monitored by subjecting aminoacyl-S-SgcC2 substrate 6 and product 10 to alkaline hydrolysis followed by HPLC analysis. A new peak appeared eluting after 4 (obtained from hydrolysis of substrate $\mathbf{6}$ ), and this new peak had an identical retention time to 
authentic $\mathbf{1 3}$ (Figure 4). The identity of the hydrolyzed product as $\mathbf{1 3}$ was confirmed by ESIMS, yielding a pair of $[\mathrm{M}+\mathrm{H}]^{+}$ions at $m / z=216.2$ and 218.1 with 3:1 ratio, characteristic for the mono-chlorinated $\mathbf{1 3}$ (molecular formula $\mathrm{C}_{9} \mathrm{H}_{10} \mathrm{O}_{3} \mathrm{NCl}$, calcd 215.0 and 217.0) (see Figure $\mathrm{S} 2$ in Supporting Information). Time course analysis showed that $\mathrm{SgcC} 3$-catalyzed the conversion of $\mathbf{6}$ to $\mathbf{1 0}$ in a time-dependent reaction with a specific activity of $0.93 \pm 0.14$ $\mathrm{hr}^{-1}$ (Figure 4).

\section{Cofactor and Co-substrate Requirements for SgcC3-Catalyzed Chlorination}

The substrate and cofactor requirement for the $\mathrm{SgcC} 3$-catalyzed chlorination of $\mathbf{6}$ to $\mathbf{1 0}$ as depicted in Figure 5A was next examined (results summarized in Table 1). Both $\mathrm{O}_{2}$ and NADH are required for $\mathrm{SgcC} 3$ catalysis - the removal of either completely abolished $\mathbf{1 0}$ formation (Figure 5B, VI and VII). When FAD was excluded from the assay solution, a minute amount of $\mathbf{1 0}$ was detected by HPLC, consistent with the observation that $\mathrm{SgcC} 3$ is co-purified with sub-stoichiometric amounts of FAD that can be utilized for $\mathrm{SgcC} 3$ catalysis (Figure 5B, V). In the absense of a flavin reductase, a small amount of $\mathbf{1 0}$ was produced, and this activity is likely due to co-purification of $\mathrm{SgcC} 3$ with $E$. coli Fre as has been reported for other halogenases 27 and flavin-dependent oxygenase. ${ }^{35,36}$ However, optimal SgcC 3 activity requires the exogenous supply of a flavin reductase (Figure 5B, IV vs II and III). Finally, there appears to be no specific interaction between $\mathrm{SgcC} 3$ and the flavin reductase since $\mathrm{SgcE} 6$ and Fre can equally support the halogenase activity of $\mathrm{SgcC} 3$ (Figure 5B, II and III). This finding is consistent with the observation that SgcE6 is the only flavin reductase within the C-1027 cluster, ${ }^{8}$ which likely serves all of the flavin-dependent enzymes involved in C-1027 biosynthesis.

\section{pH Optimum for SgcC3 Activity}

To estimate the $\mathrm{pH}$ optimum for $\mathrm{SgcC}$, chlorination of $\mathbf{6}$ was carried out in $50 \mathrm{mM}$ sodium acetate, sodium phosphate, and Tris- $\mathrm{HCl}$ buffer, ranging from $\mathrm{pH} 5.0$ to $\mathrm{pH} 9.0$, under the standard assay conditions. $\mathrm{SgcC} 3$ showed the highest activity at pH 6.0 (see Figure $\mathrm{S} 3$ in Supporting Information), and therefore all subsequent assays were performed in $50 \mathrm{mM}$ phosphate buffer at $\mathrm{pH} 6.0$.

\section{Substrate Specificity of SgcC3}

The substrate specificity of $\mathrm{SgcC} 3$ was initially examined with free amino acids as substrates, including 4, 11, and 3-hydroxy- $\beta$-tyrosine. HPLC analysis showed no activity under all conditions tested, consistent with the previous proposal that chlorination occurs after $\mathbf{4}$ is tethered to $\mathrm{SgcC} 2$ as 6 (Figure 2B). $8,10-14$

The substrate specificity of $\mathrm{SgcC} 3$ was next investigated by using a series of $\beta$-aminoacyl-S$\mathrm{SgcC} 2$ as substrates (Figure 5A and 5C). Taking advantage of SgcC1's ability to activate and load other $\beta$-amino acids to $\mathrm{SgcC} 2,{ }^{13,14} \mathbf{1 2}$ and 3-hydroxy- $\beta$-tyrosyl-SgcC2 were prepared and tested, in comparison with $\mathbf{6}$, for activity with $\mathrm{SgcC} 3$. Unexpectedly, $\mathrm{SgcC} 3$ catalyzes the chlorination of $\mathbf{1 2}$ to generate $\mathbf{1 6}$ with a specific activity of $1.1 \pm 0.20 \mathrm{hr}^{-1}$, nearly identical to that found with 6 (Figure 5C II and III). In contrast, no chlorination was detected with 3hydroxy- $\beta$-tyrosyl-SgcC2 in spite of the inclusion of a 5-fold amount of $\mathrm{SgcC} 3$ and prolonged incubation time. The latter finding is consistent with $\mathbf{6}$ as the bona fide substrate of $\mathrm{SgcC} 3$ and supports the timing of the individual steps proposed for 3 biosynthesis (Figure 2B). ${ }^{8,10-14}$

Finally, halogen specificity of $\mathrm{SgcC} 3$ was examined. After purification of all proteins in the absence of chloride, $\mathrm{SgcC} 3$ efficiently catalyzed the bromination of 6 to afford the corresponding brominated product 3-bromo- $\beta$-tyrosyl-SgcC2 (17) with a specific activity of $0.36 \pm 0.10 \mathrm{hr}^{-1}$, which is approximately 2 -fold less than chlorination under similar reaction conditions (Figure 2C, V and VI). However, neither fluorination nor iodination was observed 
under the identical condition, a finding that is consistent with all flavin-dependent halogenases known to date. ${ }^{16,22}$ The identity of $\mathbf{1 7}$ was confirmed by HPLC analysis of the free acid $\mathbf{1 5}$ using synthetic $\mathbf{1 5}$ as a standard. ESI-MS analysis of $\mathbf{1 5}$ yielded a pair of $[\mathrm{M}+\mathrm{H}]^{+}$ions at $\mathrm{m} / \mathrm{z}$ $=260.0$ and 262.0 with 1:1 ratio, in agreement with the mono-bromonated $\mathbf{1 5}$ (molecular formula $\mathrm{C}_{9} \mathrm{H}_{10} \mathrm{O}_{3} \mathrm{NBr}$, calcd 259.0 and 261.0 around 1: 1 ratio).

\section{Discussion}

Like all enediyne natural products, $\mathrm{C}-1027$ shows a potent antitumor activity. Despite sharing a common enediyne core, however, $\mathrm{C}-1027$ is unique within the family in that it is estimated to 1000-times more potent than other 9-membered enediynes such as neocarzinostatin or 10membered enediynes such as calicheamicin. ${ }^{37}$ This difference in cytotoxicity can be attributed in part to the moieties decorating the enediyne core, for which C-1027 has three - a deoxy aminosugar, a benzoxazolinate moiety, and a $\beta$-amino acid moiety (Figure 1). Indeed, as we have recently reported, small structural permutations to the benzoxazolinate and $\beta$-amino acid moieties have profound effects on the biological activity of C-1027, including the ability to induce DSB, the in vivo cytotoxicity, and the mechanism of the cellular response upon treatment with C-1027. ${ }^{9}$

The 3-chloro-4,5-dihydroxy- $\beta$-phenylalanine moiety of 1 contributes critical interactions with the apo-protein CagA and, as suggested from the studies regarding the bioactivity of $\mathbf{7}$ and $\mathbf{9}$, 9 modulates the stability and reactivity of the enediyne core via $\pi$ - $\pi$ stacking interaction. ${ }^{38}$

This moiety is activated and incorporated into C-1027 by first generating a $(S)$ - $\beta$-tyrosyl-S$\mathrm{SgcC} 2$ intermediate $\mathbf{6}$, which subsequently serves as a substrate for chlorination and likely hydroxylation prior to attachment to the enediyne core. ${ }^{8,13}$ The process of activation and incorporation is achieved by enzymes with homology to protein domains found in nonribosmal peptide synthetases (NRPS), and these enzymes $-\mathrm{SgcC} 1, \mathrm{SgcC} 2$, and $\mathrm{SgcC} 5$ - are located in a sub-clustered region within the $\sim 80 \mathrm{~kb} \mathrm{C}-1027$ biosynthetic gene cluster (Figure $2 \mathrm{~A}$ ). In contrast, a single candidate for halogenation, $\mathrm{SgcC} 3$, was located upstream and distant from the NRPS locus. It only became apparent that $\mathrm{SgcC} 3$ was involved in chlorination of the $\beta$ amino acid moiety upon inactivation of $\mathrm{sgcC} 3$ and isolation of the expected deschloro analog 7. ${ }^{13}$ To unravel the molecular details of this transformation, we cloned $\operatorname{sgcC} 3$ and overproduced and purified the recombinant protein for in vitro characterization.

Initial activity tests revealed that $\mathrm{SgcC} 3$ does not catalyze the chlorination of free amino acid substrates, including 4 . As a result, 6 was enzymatically prepared from 4 in vitro using the Svp PPTase, ${ }^{34}$ the SgcC1 adenylation enzyme, and the SgcC2 PCP, all of which have been previously characterized. ${ }^{13,14}$ After purification of the resulting aminoacyl-S-SgcC2 and prior to activity tests, hydrolysis of $\beta$-amino acids from $\mathrm{SgcC} 2$ was optimized to afford a stoichiometric release for detection by HPLC. Activity of $\mathrm{SgcC} 3$ was examined with $\mathrm{NaCl}$ as a halide donor, and a single, new peak was observed only when (i) exogenous FAD was supplied to the reaction mixture, (ii) an NADH-dependent flavin reductase was included to provide diffusible $\mathrm{FADH}_{2}$, and (iii) the reaction was performed in an aerobic environment. This new peak was confirmed to be $\mathbf{1 3}$ based on comparisons to synthetic standard and ESIMS analysis. These results provided unambiguous evidence that $\mathrm{SgcC} 3$ is a $\mathrm{FADH}_{2}, \mathrm{O}_{2^{-}}$ dependent halogenase that requires a carrier protein-tethered substrate for activity. The cofactor and co-substrate requirements are similar to those observed for the halogenase PltA involved in pyoluteorin biosynthesis, ${ }^{27}$ and these two enzymes now represent a growing family of $\mathrm{FADH}_{2}, \mathrm{O}_{2}$, and carrier protein- dependent halogenases for which several have been predicted from bioinformatics analysis. ${ }^{16}$

We previously reported that $\mathrm{SgcC} 1$ specifically activates 4 , but also activates other $\beta$-tyrosine analogues including 11, 3 -chloro- $\beta$-tyrosine, 3 -hydroxy- $\beta$-tyrosine and 3 -chloro-5-hydroxy- 
$\beta$-tyrosine, albeit with minimally 25 -fold less efficiency. ${ }^{13,14}$ This indicates that C-3 chlorination and C-5 hydroxylation of $\mathbf{4}$ occurs most likely after $\mathbf{4}$ is tethered to $\mathrm{SgcC} 2$, a prediction that is now confirmed by the finding that $\mathbf{4}$ is not a substrate for $\mathrm{SgcC} 3$. However, these results fell short of revealing any insight into the timing of halogenation after 4 is tethered to $\mathrm{SgcC} 2$. Therefore, both $\mathbf{1 2}$ and 3-hydroxy- $\beta$-tyrosyl-S-SgcC2 were enzymatically prepared by taking advantage of the promiscuous nature of $\mathrm{SgcC} 1$. Remarkably, $\mathrm{SgcC} 3$ catalyzes the chlorination of $\mathbf{1 2}$ into $\mathbf{1 6}$ with a specificity activity that is almost identical to that for $\mathbf{6}$. While this may appear to be unexpected, it in fact is consistent with the early finding that $\mathrm{SgcC} 1$ has a 25 -fold selectivity for $\mathbf{4}$ over 11, serving as the "gate-keeper" that controls the $\beta$-amino acid to be incorporated into 1 in vivo. ${ }^{13,14}$ In contrast, no chlorination was detected with 3hydroxy- $\beta$-tyrosyl-S-SgcC2, the alternative substrate for $\mathrm{SgcC} 3$ if $\mathrm{C}-5$ hydroxylation occurred prior to $\mathrm{C}-3$ halogenation. This is consistent with $\mathbf{6}$ as the preferred substrate of $\mathrm{SgcC} 3$ and further supports the timing of the individual steps proposed for $\mathbf{3}$ biosynthesis (Figure 2B). Finally, similar to other halogenases, chloride can be substituted with bromide but not fluoride or iodide, and structural analysis of $\mathrm{SgcC} 3$ should provide insights into this selectivity.

In conclusion, $\mathrm{SgcC} 3$ has been shown to catalyze the regioselective chlorination of $\mathbf{6}$, and this activity is dependent on $\mathrm{O}_{2}$ and $\mathrm{FADH}_{2}$. The requirements for $\mathrm{SgcC} 3$ catalysis are consistent with the catalytic cycle proposed for $\mathrm{FADH}_{2}, \mathrm{O}_{2}$-dependent halogenases as depicted in Figure 5A. The fact that recombinant strains of $S$. globisporus can produce $\mathrm{C}-1027$ analogs $\mathbf{7}, \mathbf{8}$, and 9 suggests enzymes downstream of $\mathrm{SgcC} 3$ have relaxed substrate specificity. Along with the results for $\mathrm{SgcC} 3$ provided here, application of precursor-directed biosynthesis and combinatorial biosynthesis methods to the $\mathrm{C}-1027$ biosynthetic machinery presents a unique opportunity to generate novel C-1027 analogs some of which could have improved biological activity.

\section{Supplementary Material}

Refer to Web version on PubMed Central for supplementary material.

\section{Acknowledgements}

We thank Dr. Y. Li, Institute of Medicinal Biotechnology, Chinese Academy of Medical Sciences, Beijing, China for the wild-type S. globisporus strain and the Analytical Instrumentation Center of the School of Pharmacy, University of Wisconsin-Madison for support in obtaining MS and NMR data. This work is supported in part by NIH grants CA78747 and CA113297. SVL is the recipient of an NIH postdoctoral fellowship (CA1059845).

\section{Abbreviations used}

CoA

coenzyme A

DSB

double-stranded breaks

ESI-MS

electrospray ionization-mass spectrometry

FAD

flavin adenine dinucleotide

FMN

flavin adenine mononucleotide

FADH $_{2}$

reduced flavin adenine dinucleotide 


\section{NADH}

$\beta$-nicotinamide adenine dinuceotide, reduced

NRPS

nonribosomal peptide synthetase

PCP

peptidyl carrier protein

PPTase

phosphopantetheinyl transferase

TCEP

tris(2-carboxyethyl)phosphine hydrochloride

\section{References}

1. Hu J, Xue YC, Xie MY, Zhang R, Otani T, Minami Y, Yamada Y, Marunaka T. J Antibiot 1988;41:1575-1579. [PubMed: 3198491]

2. Maeda, H.; Edo, K.; Ishida, N., editors. Drug. Springer; Tokyo: 1997. Neocarzinostatin: the Past, Present, and Future of an Anticancer.

3. Schroeder DR, Closon KL, Klohr SE, Zein N, Langley DR, Lee MS, Matson JA, Doyle TW. J Am Chem Soc 1994;116:9351-9352.

4. Otani T, Yasuhara T, Minami Y, Shimazu T, Zhang R, Xie MY. Agric Biol Chem 1991;55:407-417. [PubMed: 1368692]

5. Sugimoto Y, Otani T, Oie S, Wierzba K, Yamada Y. J Antibiot 1990;43:417-421. [PubMed: 2161819]

6. Sugiura Y, Matsumoto T. Biochemistry 1993;32:5548-5553. [PubMed: 8504075]

7. Xu Y, Zhen Y, Zhen Y, Goldberg IH. Biochemistry 1995;34:12451-12460. [PubMed: 7547991]

8. Liu W, Christenson SD, Standage S, Shen B. Science 2002;297:1170-1173. [PubMed: 12183628]

9. Kennedy DR, Gawron LS, Ju J, Liu W, Shen B, Beerman TA. Cancer Res 2007;67:773-781. [PubMed: 17234789]

10. Christenson SD, Liu W, Toney MD, Shen B. J Am Chem Soc 2003;125:6062-6063. [PubMed: 12785829]

11. Christenson SD, Wu W, Spies MA, Shen B, Toney MD. Biochemistry 2003;42:12708-12718. [PubMed: 14580219]

12. Christianson CW, Montavon TJ, Van Lanen SG, Shen B, Bruner SD. Biochemistry 2007;46:72057214. [PubMed: 17516659]

13. Van Lanen SG, Dorrestein PC, Christenson SD, Liu W, Ju J, Kelleher NL, Shen B. J Am Chem, Soc 2005;127:11594-11595. [PubMed: 16104723]

14. Van Lanen SG, Lin S, Dorrestein PC, Kelleher NL, Shen B. J Biol Chem 2006;281:29633-29640. [PubMed: 16887797]

15. Van Pee KH. Arch Microbiol 2001;175:250-258. [PubMed: 11382220]

16. Vaillancourt FH, Yeh E, Vosburg DA, Garneau-Tsodikova S, Walsh CT. Chem Rev 2006;106:33643378. [PubMed: 16895332]

17. Vaillancourt FH, Yeh E, Vosberg DA, O’Connor SE, Walsh CT. Nature 2005;436:1191-1194. [PubMed: 16121186]

18. Kelly WL, Boyne MT II, Yeh E, Vosburg DA, Galonic DP, Kelleher NL, Walsh CT. Biochemistry 2007;46:359-368. [PubMed: 17209546]

19. Vaillancourt FH, Yin J, Walsh CT. Proc Natl Acad Sci US A 2005;102:10111-10116.

20. Blasiak LC, Vaillancourt FH, Walsh CT, Drennan CL. Nature 2006;440:368-371. [PubMed: 16541079]

21. Galonic DP, Vaillancourt FH, Walsh CT. J Am Chem Soc 2006;128:3900-3901. [PubMed: 16551084]

22. Van Pee KH, Patallo EP. Appl Microbiol Biotech 2006;70:631-641. 
23. Zehner S, Kotzsch A, Bister B, Sussmuth RD, Mendez C, Salas JA, Van Pee KH. Chem Biol 2005;12:445-452. [PubMed: 15850981]

24. Keller S, Wage T, Hohaus K, Holzer M, Eichhorn E, Van Pee KH. Angew Chem Int Ed 2000;39:23002302.

25. Dong C, Flecks S, Unversucht S, Haupt C, Van Pee KH, Naismith JH. Nature 2005;309:2216-2219.

26. Yeh E, Garneau S, Walsh CT. Proc Natl Acad Sci USA 2005;102:3960-3965. [PubMed: 15743914]

27. Dorrestein PC, Yeh E, Garneau-Tsodikova S, Kelleher NL, Walsh CT. Proc Natl Acad Sci USA 2005;102:13843-13848. [PubMed: 16162666]

28. Yeh E, Cole LJ, Barr EW, Bollinger JM, Ballou DP, Walsh CT. Biochemistry 2006;45:7904-7912. [PubMed: 16784243]

29. Yeh E, Blasiak LC, Koglin A, Drennan CL, Walsh CT. Biochemistry 2007;46:1284-1292. [PubMed: 17260957]

30. Holzer M, Burd W, Reissig HU, van Pee KH. Adv Synth Catal 2001;343:591-595.

31. Wynands I, van Pee KH. FEMS Microbiol Lett 2004;237:363-367. [PubMed: 15321684]

32. Tan CYK, Weaver DF. Tetrahedron 2002;58:7449-7461.

33. Sambrook, J.; Fritsch, EF.; Maniatis, T. Molecular cloning: A Laboratory Mannual. 3. Cold Spring Harbor Laboratory Press; Cold Spring Harbor, NY: 2000.

34. Sanchez C, Du LC, Edwards DJ, Toney MD, Shen B. Chem Biol 2001;8:725-738. [PubMed: 11451672]

35. Eichhorn E, van de Ploeg JR, Leisinger T. J Biol Chem 1999;274:26639-26646. [PubMed: 10480865]

36. Fieschi F, Niviere V, Frier C, Decout JL, Fontecave M. J Biol Chem 1995;270:30392-30400. [PubMed: 8530465]

37. Wang XW, Hong X. Drugs of the Future 1999;24:847-852.

38. Okuno Y, Otsuka M, Sugiura Y. J Med Chem 1994;37:2266-2273. [PubMed: 8057275]

36. van Berkel WJH, Kamerbeek NM, Fraaije MW. J Biotech 2006;124:670-689.

37. Orser CS, Lange CC, Xun L, Zahrt TC, Schneider BJ. J Bacteriol 1993;175:411-416. [PubMed: 7678243] 


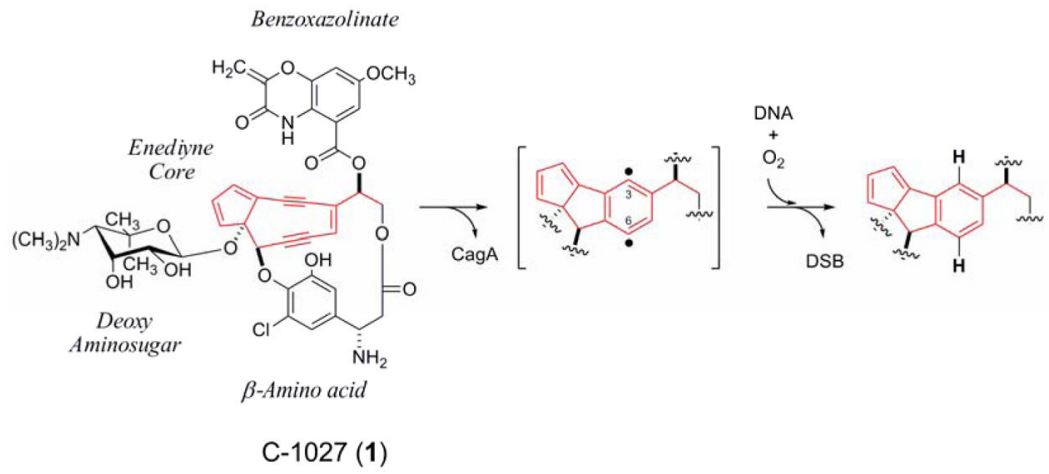

Figure 1.

The Bergman cycloaromatization exemplified by the C-1027 chromophore. Upon release from the apo-protein CagA, the enediyne core (shown in red) undergoes an electronic rearrangement to form a 3, 6-diradical species that can abstract hydrogen atoms from DNA. DSB, doublestranded break. 
A

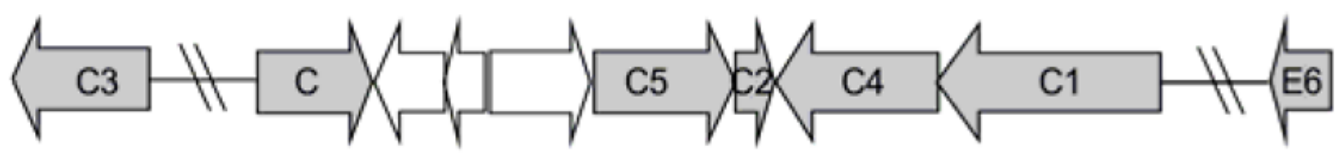

B<smiles>COC(=O)CC(N)c1ccc(O)cc1</smiles>

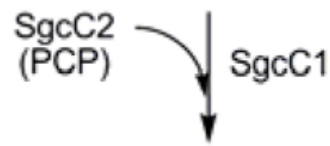

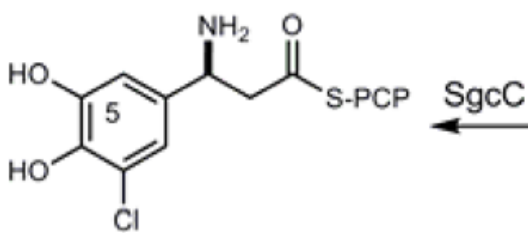

3<smiles>NC(CC(=O)S[Pb])c1ccc(O)c(Cl)c1</smiles>

10<smiles>C1[As][Se][Se]1</smiles>

$\mathrm{SgcC} 3$ SgcE6

6

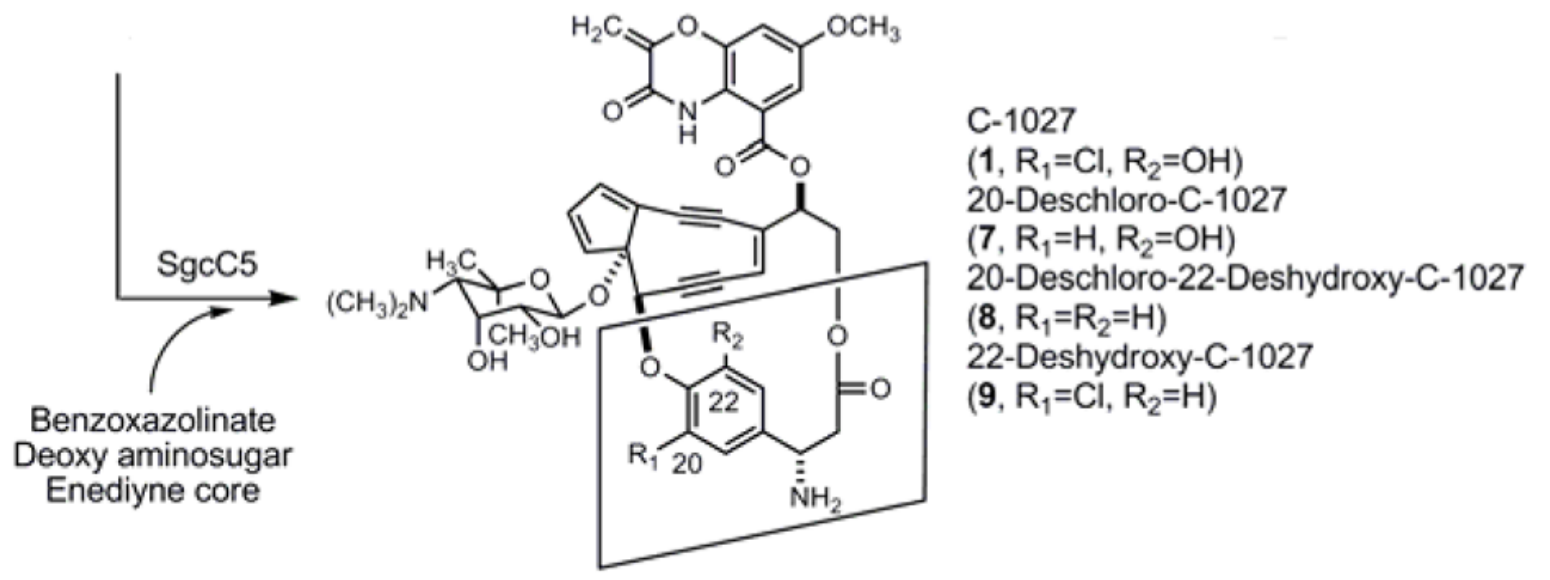

Figure 2.

Biosynthesis of (S)-3-chloro-4,5-dihydroxy- $\beta$-phenylalanine moiety (3) of C-1027 (1, boxed). (A) A sub-cluster of genes within the C-1027 gene cluster that encode biosynthetic proteins for $\mathbf{3}$ production and (B) proposed biosynthetic pathway for $\mathbf{3}$ from L- $\alpha$-tyrosine (2) and structures of $\mathbf{1}$ and engineered analogs $\mathbf{7}, \mathbf{8}$, and $\mathbf{9}$. 


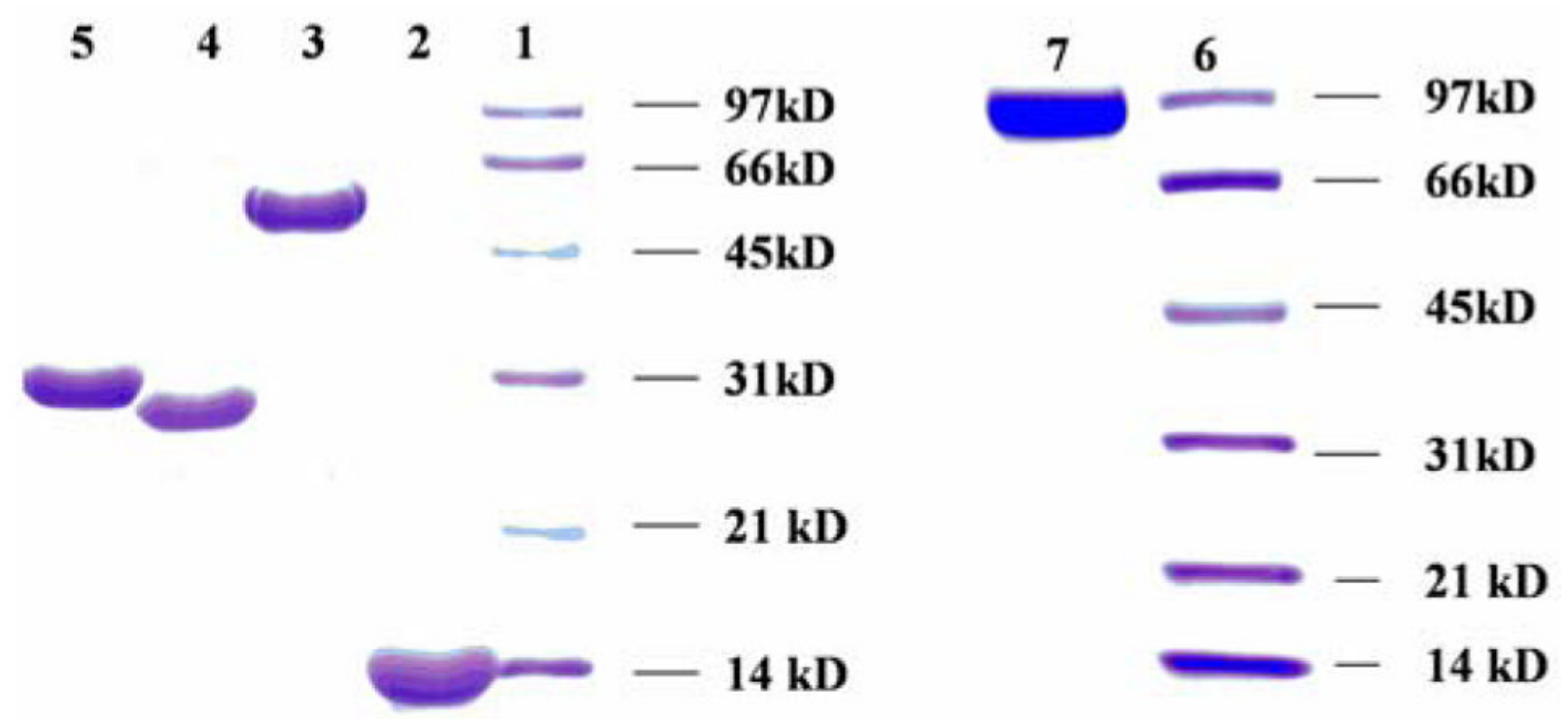

Figure 3.

Purification and characterization of proteins used in this study as judged by SDS-PAGE: lane 1, SgcC2; lane 3, SgcC3; lane 4, SgcE6; lane 5, Fre; lane 7, SgcC1; lanes 1 and 6, low range molecular weight standards. 


\section{$\mathrm{AU}$ at $280 \mathrm{~nm}$}

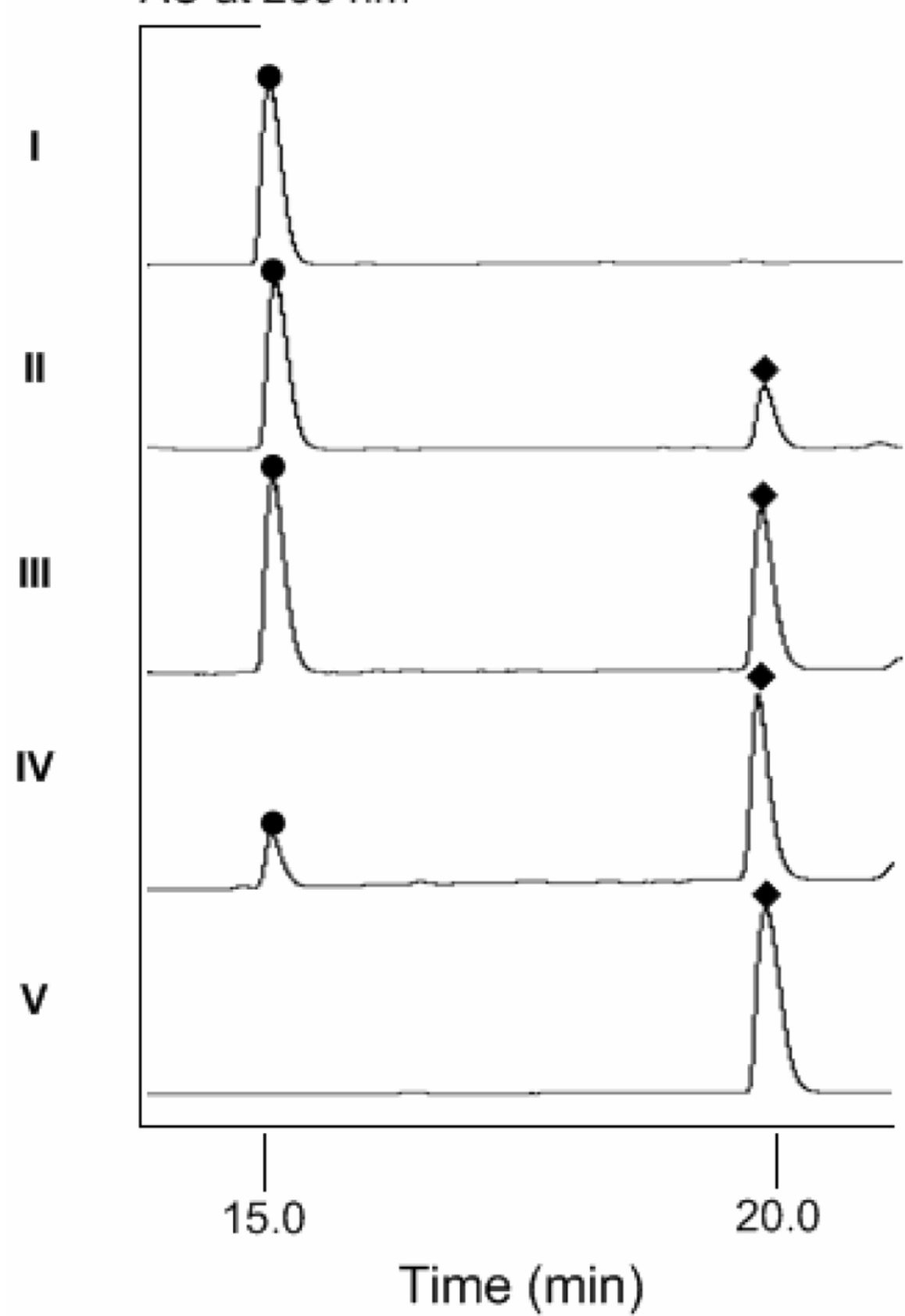

Figure 4.

HPLC chromatograms of $\mathrm{SgcC} 3$ assays showing authentic $(S)$ - $\beta$-tyrosine (4) standard (I), incubation of $(S)$ - $\beta$-tyrosyl-S-SgcC2 (6) with $\mathrm{SgcC} 3$ at $37^{\circ} \mathrm{C}$ for $15 \mathrm{~min}$ (II), $30 \mathrm{~min}$ (III), 60 $\min (\mathrm{IV})$, and synthetic 3 -choro- $\beta$-tyrosine (V). $(\bullet), \mathbf{4}$ and $(\diamond), \mathbf{1 3}$ or 3 -choro- $\beta$-tyrosine. 
A
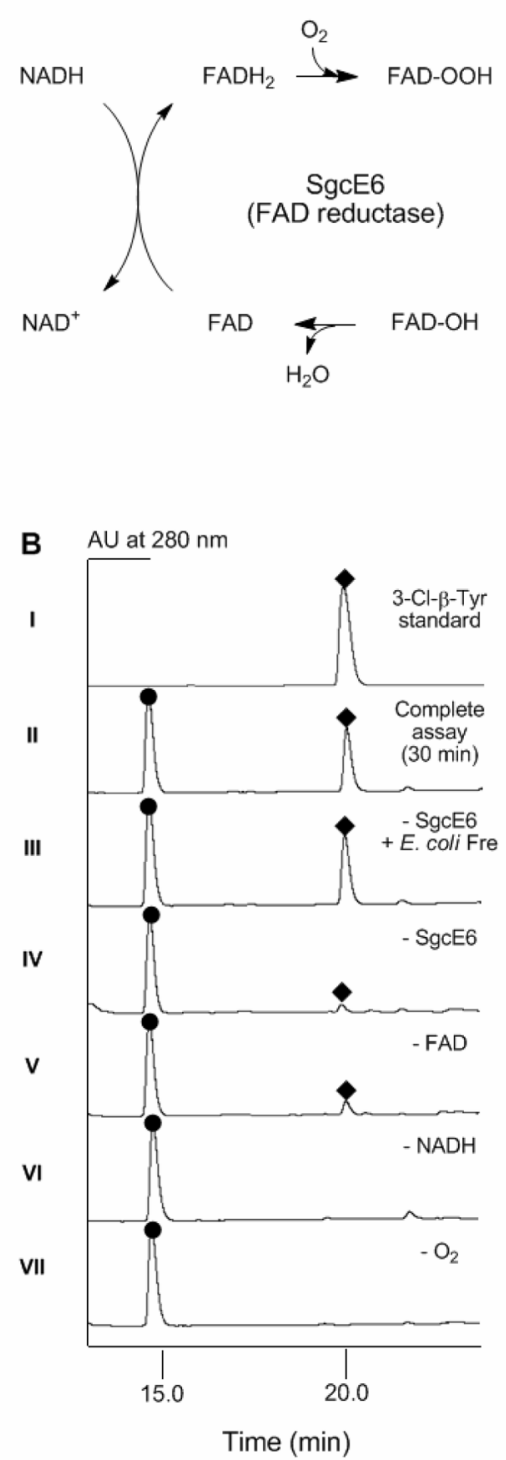
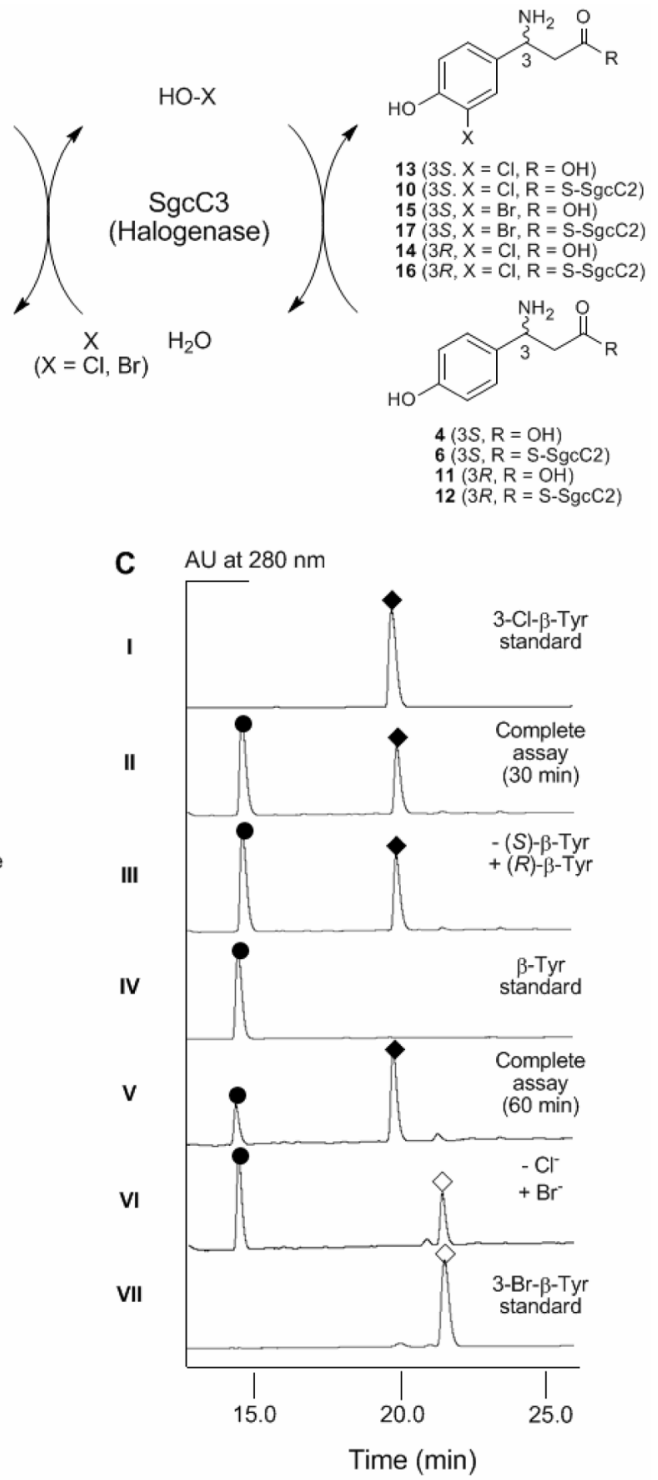

Figure 5.

SgcC3-catalyzed halogenation of $(S)$ - $\beta$-tyrosyl-S-SgcC2 (6) or $(R)$ - $\beta$-tyrosyl-S-SgcC2 (11). (A) The reaction scheme for $\mathrm{SgcC} 3$ depicting a catalytic cycle involving the FAD reductase SgcE6. (B) HPLC chromatograms examining substrate and cofactor requirement for SgcC3catalyzed chlorination of $\mathbf{6}$. (C) HPLC chromatograms examining alternative substrates for SgcC3. $(\bullet), 4$ or $11 ;(\diamond), 13,14$ or 3 -choro- $\beta$-tyrosine; $(\diamond), 15$ or 3-bromo- $\beta$-tyrosine. 
Table 1

Cofactor and co-substrate requirement for SgcC3-catalyzed chlorination of $(S)$ - $\beta$-tyrosyl-S-SgcC2 (6) in vitro.

\begin{tabular}{cccc}
\hline Entry $^{\boldsymbol{a}}$ & Condition & Conversion \\
\hline II & Complete assay & $38 \%$ & 100 \\
III & Complete assay - SgcE6 + E. Coli Fre & $39 \%$ & $6 \%$ \\
IV & Complete assay - SgcE6 & $11 \%$ & 0 \\
V & Complete assay - FAD & 0 & 0 \\
VI & Complete assay - NADH & 0 & 0 \\
VII & Complete assay - $\mathrm{O}_{2}$ & 0 \\
\hline
\end{tabular}

${ }^{a}$ Entry number corresponds to the chromatogram shown in Figure 5B. 